\title{
Bimodal or quadrimodal? Statistical tests for the shape of fault patterns
}

\author{
David Healy ${ }^{1}$ and Peter Jupp ${ }^{2}$ \\ ${ }^{1}$ School of Geosciences, King's College, University of Aberdeen, Aberdeen AB24 3UE Scotland, UK \\ ${ }^{2}$ School of Mathematics \& Statistics, University of St Andrews, St Andrews KY16 9SS Scotland, UK
}

Correspondence: David Healy (d.healy@abdn.ac.uk)

Received: 25 April 2018 - Discussion started: 2 May 2018

Revised: 6 August 2018 - Accepted: 7 August 2018 - Published: 22 August 2018

\begin{abstract}
Natural fault patterns formed in response to a single tectonic event often display significant variation in their orientation distribution. The cause of this variation is the subject of some debate: it could be "noise" on underlying conjugate (or bimodal) fault patterns or it could be intrinsic "signal" from an underlying polymodal (e.g. quadrimodal) pattern. In this contribution, we present new statistical tests to assess the probability of a fault pattern having two (bimodal, or conjugate) or four (quadrimodal) underlying modes and orthorhombic symmetry. We use the eigenvalues of the second- and fourth-rank orientation tensors, derived from the direction cosines of the poles to the fault planes, as the basis for our tests. Using a combination of the existing fabric eigenvalue (or modified Flinn) plot and our new tests, we can discriminate reliably between bimodal (conjugate) and quadrimodal fault patterns. We validate our tests using synthetic fault orientation datasets constructed from multimodal Watson distributions and then assess six natural fault datasets from outcrops and earthquake focal plane solutions. We show that five out of six of these natural datasets are probably quadrimodal and orthorhombic. The tests have been implemented in the $\mathrm{R}$ language and a link is given to the authors' source code.
\end{abstract}

\section{Introduction}

\subsection{Background}

Faults are common structures in the Earth's crust, and they rarely occur in isolation. Patterns of faults, and other fractures such as joints and veins, control the bulk transport and mechanical properties of the crust. For example, arrays of low-permeability (or "sealing") faults in a rock matrix of higher permeability can produce anisotropy of permeability and preferred directions of fluid flow. Arrays of weak faults can similarly produce anisotropy, i.e. directional variations, of bulk strength. It is important to understand fault patterns, and quantifying the geometrical attributes of any pattern is an important first step. Faults, taken as a class of brittle shear fractures, are often assumed to form in conjugate arrays, with fault planes more or less evenly distributed about the largest principal compressive stress, $\sigma_{1}$, and making an acute angle with it. This model, an amalgam of theory and empirical observation, predicts that conjugate fault planes intersect along the line of $\sigma_{2}$ (the intermediate principal stress) and the fault pattern overall displays bimodal symmetry (Fig. 1a). A fundamental limitation of this model is that these fault patterns can only ever produce a plane strain (intermediate principal strain $\varepsilon_{2}=0$ ), with no extension or shortening in the direction of $\sigma_{2}$. This kinematic limitation is inconsistent with field and laboratory observations that document the existence of polymodal or quadrimodal fault patterns and which produce triaxial strains in response to triaxial stresses (e.g. Aydin and Reches, 1982; Reches, 1978; Blenkinsop, 2008; Healy et al., 2015; McCormack and McClay, 2018). Polymodal and quadrimodal fault patterns possess orthorhombic symmetry (Fig. $1 \mathrm{~b}$ and c).

Fault patterns are most often visualised through maps of their traces and equal-angle (stereographic) or equal-area projections of poles to fault planes or great circles. Azimuthal projection methods (hereafter "stereograms") provide a measure of the orientation distribution, including the attitude and the shape of the overall pattern. However, these plots can be unsatisfactory when they contain many data points or 


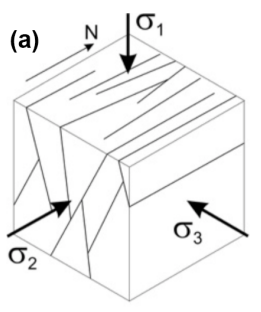

Bimodal, or conjugate (d)

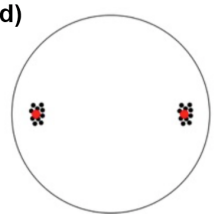

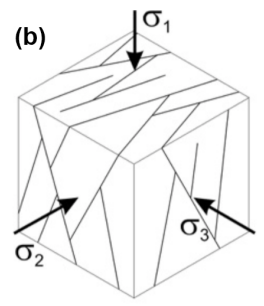

(e)

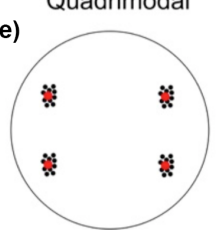

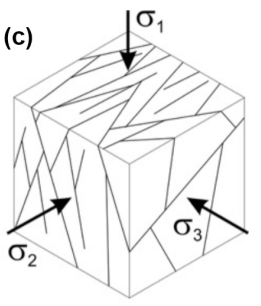

(f)

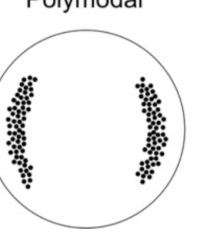

Figure 1. Schematic diagrams to compare conjugate fault patterns displaying bimodal symmetry with quadrimodal and polymodal fault patterns displaying orthorhombic symmetry. (a-c) Block diagrams showing patterns of normal faults and their relationship to the principal stresses. (d-f) Stereographic projections (equal area, lower hemisphere) showing poles to fault planes for the models shown in (a)-(c). Natural examples of all three patterns have been found in naturally deformed rocks.

the data are quite widely dispersed. Woodcock (1977) developed the idea of the fabric shape, based on the fabric or orientation tensor of Scheidegger (1965). The eigenvalues of this second-rank tensor can be used in a modified Flinn plot (Flinn, 1962; Ramsay, 1967) to discriminate between clusters and girdles of poles. These plots can be useful for three of the five possible fabric symmetry classes - spherical, axial and orthorhombic - because the three fabric eigenvectors coincide with the three symmetry axes. However, there are issues with the interpretation of distributions that are not uniaxial (Woodcock, 1977). We address these issues in this paper. Reches (Reches, 1978, 1983; Aydin and Reches, 1982; Reches and Dieterich, 1983) has exploited the orthorhombic symmetry of measured quadrimodal fault patterns to explore the relationship between their geometric-kinematic attributes and tectonic stress. More recently, Yielding (2016) measured the branch lines of intersecting normal faults from seismic reflection data and found they aligned with the bulk extension direction - a feature consistent with their formation as polymodal patterns. Bimodal (i.e. conjugate) fault arrays have branch lines aligned perpendicular to the bulk extension direction.

\subsection{Rationale}

The fundamental underlying differences in the symmetries of the two kinds of fault pattern - (i) bimodal and bilateral or (ii) and polymodal and orthorhombic - suggest that we should test for this symmetry using the orientation distributions of measured fault planes. The results of such tests may provide further insight into the kinematics and/or dynamics of the fault-forming process. This paper describes new
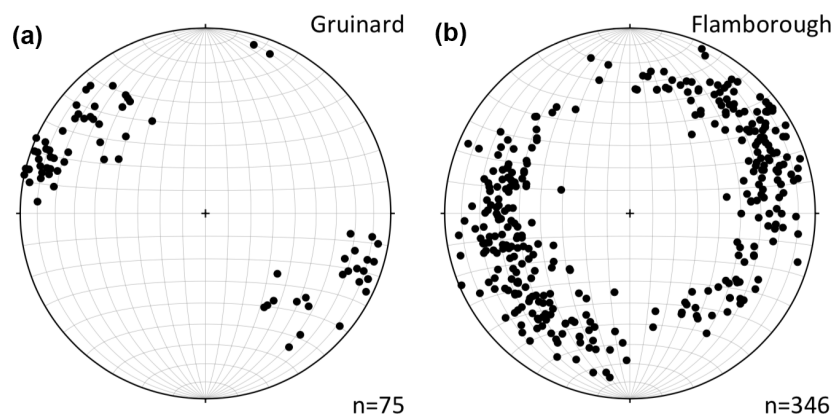

Figure 2. Stereographic projections (equal area, lower hemisphere) showing two natural fault datasets. (a) Poles to deformation bands (small offset faults; $n=75$ ) measured in Triassic sandstones at Gruinard Bay, NW Scotland (Healy et al., 2006a, b). These data were collected from a small contiguous outcrop approximately $10 \mathrm{~m}^{2}$ in area. (b) Poles to faults measured in Cretaceous chalk at Flamborough Head, NE England $(n=346)$. These data have been taken from a figure published in Peacock and Sanderson (1992) and replotted in the same format as those from Gruinard.

tests for fault pattern orientation data and includes the programme code for each test written in the $\mathrm{R}$ language ( $\mathrm{R}$ Core Team, 2017). The paper is organised as follows: Sect. 2 reviews the kinematic and mechanical issues raised by conjugate and polymodal fault patterns, in particular the implications for their orientation distributions. Section 3 describes the datasets used in this study, including synthetic and natural fault orientation distributions. Section 4 presents tests for assessing whether an orientation distribution has orthorhombic symmetry, including a description of the mathematics and the $\mathrm{R}$ code. The examples used include synthetic orientation datasets of known attributes (with and without added "noise") and natural datasets of fault patterns measured in a range of rock types. A discussion of the issues raised is provided in Sect. 5 and is followed by a short summary. The $\mathrm{R}$ code is available from http://www.mcs.st-andrews.ac.uk/ $\sim$ pej/2mode_tests.html (last access: 17 August 2018).

\section{Bimodal (conjugate) versus quadrimodal fault patterns}

Conjugate fault patterns should display bimodal or bilateral symmetry in their orientation distributions on a stereogram and ideally show evidence of central tendency about these two clusters (Fig. 1d; Healy et al., 2015). Quadrimodal fault patterns should show orthorhombic symmetry and, ideally, evidence of central tendency about the four clusters of poles on stereograms (Fig. 1e). More general polymodal patterns should show orthorhombic symmetry with an even distribution of poles in two arcs (Fig. 1f). For data collected from natural fault planes some degree of intrinsic variation, or "noise", is to be expected. Two natural example datasets are shown in Fig. 2. The Gruinard dataset is from a small area 

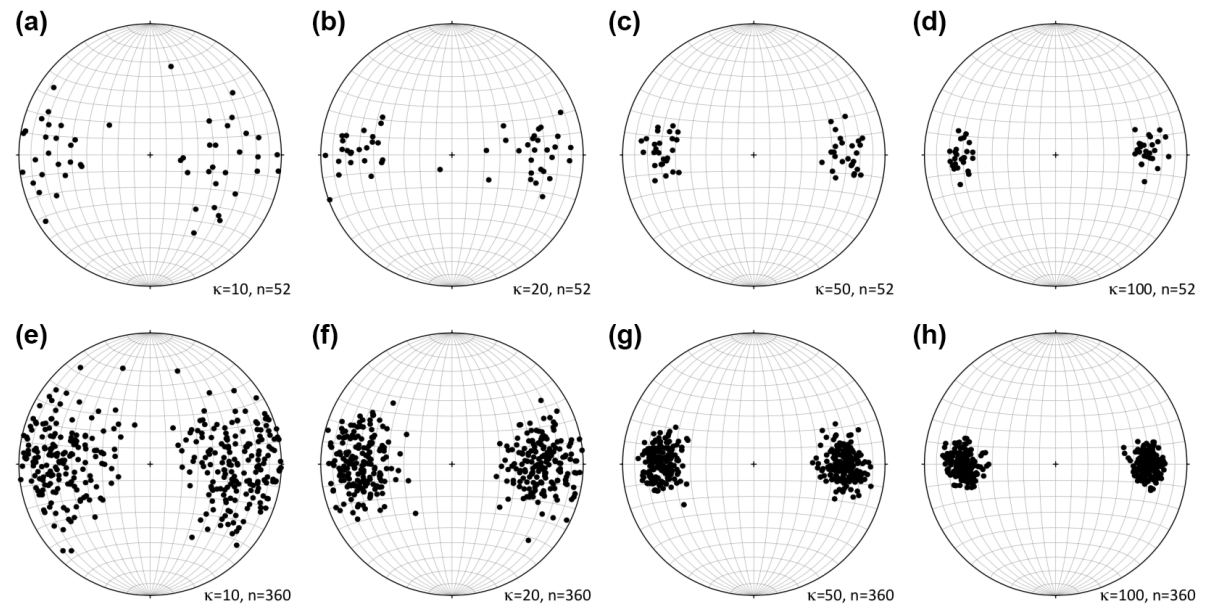

Figure 3. Stereographic projections (equal area, lower hemisphere) showing the eight synthetic datasets designed to model conjugate (bimodal) fault patterns in this study. (a-d) Synthetic fault datasets derived from equal mixtures of two Watson distributions with mean pole directions separated by an inter-fault dip angle of $60^{\circ}$. These models represent a "low fault count" scenario, with $n=52$ and $\kappa$ (the Watson dispersion parameter) varying from 10 to 100 . (e-h) These models represent a "high fault count" scenario, with $n=360$ and $\kappa$ varying from 10 to 100 .
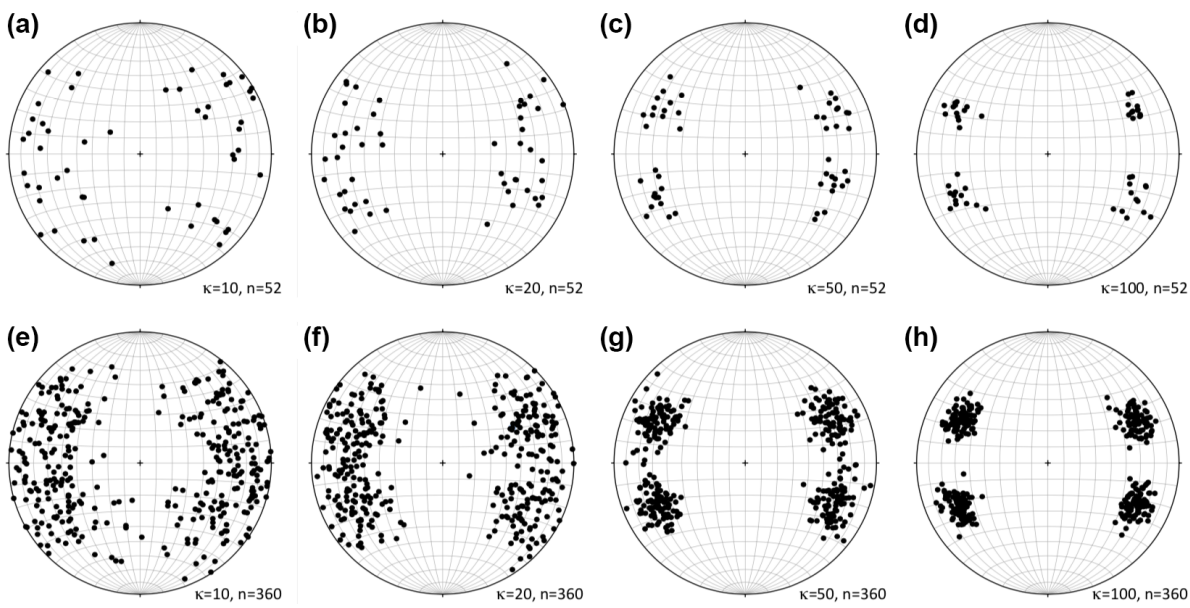

Figure 4. Stereographic projections (equal area, lower hemisphere) showing the eight synthetic datasets designed to model quadrimodal fault patterns in this study. (a-d) Synthetic fault datasets derived from equal mixtures of four Watson distributions with mean pole directions separated by an inter-fault dip angle of $60^{\circ}$ and a strike separation of $52^{\circ}$. These models represent a "low fault count" scenario, with $n=52$ and $\kappa$ (the Watson dispersion parameter) varying from 10 to 100. (e-h) These models represent a "high fault count" scenario, with $n=360$ and $\kappa$ varying from 10 to 100 .

$\left(\sim 5 \mathrm{~m}^{2}\right)$ in one outcrop of Triassic sandstone and shows poles to deformation bands with small normal offsets (millimetres to centimetres). The Flamborough dataset is taken from Peacock and Sanderson (1992; their Fig. 2a) and shows poles to normal faults in the Cretaceous chalk along a coastline section of about $1.8 \mathrm{~km}$. The authors clearly state that the approximately E-W orientation of the coastline may have generated a sampling bias in the measured data (i.e. a relative under-representation of $\mathrm{E}-\mathrm{W}$-oriented fault planes). Both datasets illustrate the nature of the problem addressed in this paper: given variable, incomplete and noisy data of different sample sizes, how can we assess the symmetry of the underlying fault pattern?

\section{Datasets used in this study}

\subsection{Synthetic datasets}

We use two sets of synthetic data to test our new statistical methods, both based on the Watson orientation distribution (Fisher et al., 1987 Sect. 4.4.4; Mardia and Jupp, 2000 Sect. 9.4.2). This is the simplest non-uniform distribution for 

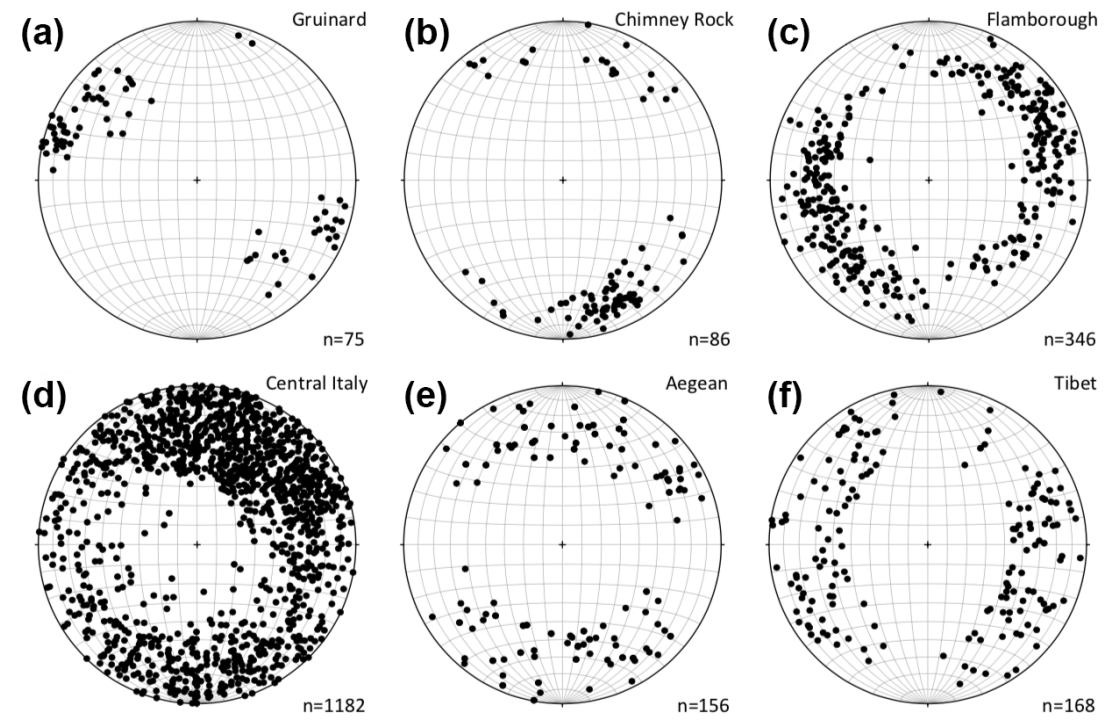

Figure 5. Stereographic projections (equal area, lower hemisphere) showing the six natural datasets used in this study. All plots show poles to faults, the majority of which are inferred to be normal. (a) Data from deformation bands measured in faulted Triassic sandstones at Gruinard Bay, Scotland (Healy et al., 2006a, b). (b) Data from faults and measured in sandstones at Chimney Rock in the San Rafael Swell, Utah, USA. Data digitised from Krantz (1989). (c) Data from faults measured in cliffs of Cretaceous chalk at Flamborough Head, NE England. Data digitised from Peacock and Sanderson (1992). (d) Data from faults measured in the Apennines of central Italy. Data digitised from Roberts (2007). (e) Data from focal mechanism nodal planes derived from the CMT catalogue for the Aegean region (Ekström et al., 2012). (f) Data from focal mechanism nodal planes derived from the CMT catalogue for the Tibet region (Ekström et al., 2012).

(a)

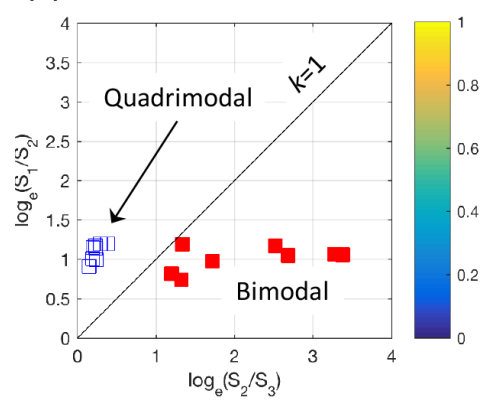

(b)

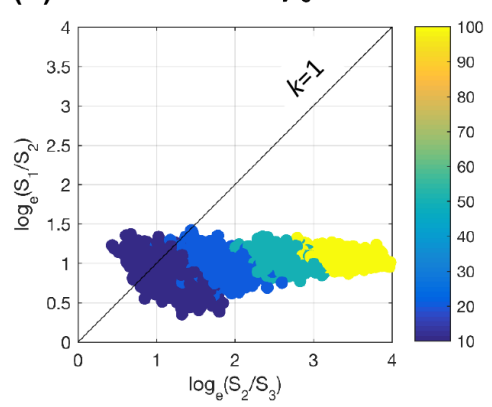

(c)

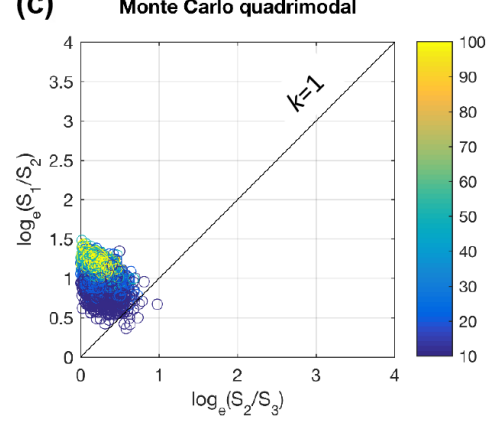

Figure 6. Graphs showing the ratios of eigenvalues of the orientation matrices for the synthetic datasets (Flinn, 1962; Ramsay, 1967; Woodcock, 1977). (a) Synthetic conjugate (i.e. bimodal; filled red symbols) and quadrimodal (hollow blue symbols) fault data. Note that the conjugate and quadrimodal data lie on either side of the line $k=1$, where $k=\log _{e}\left(S_{1} / S_{2}\right) / \log _{e}\left(S_{2} / S_{3}\right)$. (b) Eigenvalue ratios from a Monte Carlo simulation of conjugate fault orientations using the two Watson mixture model; 1000 simulations were run for each of four different $\kappa$ values (10, 20, 50 and 100; a total of 4000 data points) corresponding to the range of the discrete datasets shown in (a). (c) Eigenvalue ratios from a Monte Carlo simulation of quadrimodal fault orientations using the four Watson mixture model; 1000 simulations were run for each of four different $\kappa$ values $(10,20,50$ and 100; a total of 4000 data points) corresponding to the range of the discrete datasets shown in (a).

describing undirected lines, and has probability density

$f( \pm \boldsymbol{x} ; \boldsymbol{\mu}, \kappa) \propto \exp \left\{\kappa\left(\boldsymbol{\mu}^{T} \boldsymbol{x}\right)^{2}\right\}$

where $\kappa$ is a measure of concentration (low $\kappa$ is dispersed, high $\kappa$ is concentrated) and $\mu$ is the mean direction. To obtain a synthetic conjugate fault pattern dataset of size $n$ we combined two datasets of size $n / 2$, each from a Watson distribution, the two mean directions being separated by $60^{\circ}$.
We generated synthetic bimodal datasets with $\kappa=10,20,50$ and 100 and $n=52$ and 360 (Fig. 3). This variation in $\kappa$ provides a useful range of concentrations encompassing those observed in measured natural data and can be considered as a measure of "noise" within the distribution. Many natural datasets are often small due to limitations of outcrop size, and the two sizes of synthetic distribution ( $n=52$ and 360) allow for this fact. For synthetic polymodal fault patterns, we generated quadrimodal datasets of size $n$ by combining 
four Watson distributions of size $n / 4$ with their mean directions separated by $60^{\circ}$ in dip (as above) and $52^{\circ}$ in strike (see Healy et al., 2006a, b). By varying $n$ from 52 to 360 we cater for comparisons with smaller and larger natural datasets; as for the synthetic bimodal datasets, we varied $\kappa$ in the range of 10, 20, 50 and 100 (Fig. 4).

\subsection{Natural datasets}

We use six natural datasets of fault plane orientations from regions that have undergone or are currently undergoing extension; i.e. we believe the majority of these faults display normal kinematics (Fig. 5). The Gruinard dataset (Fig. 5a) is from Gruinard Bay in NW Scotland (UK) and featured in previous publications (Healy et al., 2006a, b). The most important thing about this dataset is that the fault planes were all measured from a small area $\left(\sim 5 \mathrm{~m}^{2}\right)$ of contiguous outcrop of a single sandstone bed. This means it is highly unlikely that the orientation data are affected by any local stress variations and subsequent possible rotations. The data were measured in normal-offset deformation bands with displacements of a few millimetres to centimetres. The next three datasets have been digitised from published papers on normal faults in Utah (Fig. 5b; Chimney Rock; Krantz, 1989), northern England (Fig. 5c; Flamborough; Peacock and Sanderson, 1992) and Italy (Fig. 5d; central Italy; Roberts, 2007). In each case, the published stereograms were digitised to extract Cartesian $(x, y)$ coordinates of the poles to faults, and these were then converted to plunge and plunge direction using the standard equations for the projection used (e.g. Lisle and Leyshon, 2004). Slight differences in the number of data plotted for each of these three with respect to the original publication arise due to the finite resolution of the digitised image of the stereograms. The last two datasets for the Aegean and Tibet (Fig. 5e and f) are derived from earthquake focal mechanisms using the CMT catalogue (Ekström et al., 2012). In each case the steepest-dipping nodal plane was selected in the absence of convincing evidence for low-angle normal faulting in these regions.

\section{Testing for orthorhombicity}

\subsection{Eigenvalue fabric (modified Flinn) plots}

We calculated the second-rank orientation tensor (Woodcock, 1977) for each of the synthetic datasets shown in Figs. 3 and 4 (bimodal and quadrimodal, respectively). The eigenvalues of this tensor $\left(S_{1}, S_{2}\right.$ and $S_{3}$, where $S_{1}$ is the largest and $S_{3}$ is the smallest) are used to plot the data on a modified Flinn diagram (Fig. 6), with $\log _{e}\left(S_{2} / S_{3}\right)$ on the $x$ axis and $\log _{e}\left(S_{1} / S_{2}\right)$ on the $y$ axis. The points corresponding to the bimodal (shown in red) and quadrimodal (shown in blue) datasets lie in distinct areas. Bimodal (conjugate) fault patterns lie below the $1: 1$ line, on which $S_{1} / S_{2}=S_{2} / S_{3}$. This is due to the $S_{3}$ eigenvalue being very low (near 0 ) for these distributions, which for high values of $\kappa$ begin to resemble girdle fabric patterns confined to the plane of the eigenvectors corresponding to eigenvalues $S_{1}$ and $S_{2}$ (Woodcock, 1977). In contrast, the quadrimodal patterns lie above the $1: 1$ line, as $S_{3}$ for these distributions is large relative to the equivalent bimodal pattern (i.e. for the same values of $\kappa$ and $n$ ). The modified Flinn plot therefore provides a potentially rapid and simple way to discriminate between bimodal (conjugate) and quadrimodal fault patterns. Note, however, that the spread of the bimodal patterns in Fig. 6a along the $x$ axis is a function of the $\kappa$ value of the underlying Watson distribution, with low values of $\kappa-$ low concentration, highly dispersed - lying closer to the origin. Dispersed or noisy bimodal (conjugate) patterns may therefore lie closer to quadrimodal patterns (see the discussion below).

\subsection{Randomisation tests using second- and fourth-rank orientation tensors}

\subsubsection{Underlying distributions}

To get a suitable general setting for our tests, we formalise the construction of the bimodal and quadrimodal datasets considered in Sect. 3.1. Whereas the datasets considered in Sect. 3.1 necessarily have equal numbers of points around each mode, for datasets arising from the distributions here, this is true only on average. The very restrictive condition of having a Watson distribution around each mode is relaxed here to that of having a circularly symmetric distribution around each mode.

Suppose that axes $\pm x_{1}, \ldots \pm x_{n}$ are independent observations from some distribution of axes. If the parent distribution is thought to be multimodal, then two appealing models are the following.

i. The bimodal equal mixture model can be thought of intuitively as obtained by "pulling apart" a unimodal distribution into two equally strong modes, angle $\alpha$ apart. More precisely, the probability density is

$$
\begin{aligned}
& f_{2}\left( \pm \boldsymbol{x} ;\left\{ \pm \boldsymbol{\mu}_{1}, \pm \boldsymbol{\mu}_{2}\right\}\right)=\frac{1}{2}\left\{g\left( \pm \boldsymbol{x} ; \pm \boldsymbol{\mu}_{1}\right)\right. \\
& \left.\quad+g\left( \pm \boldsymbol{x} ; \pm \boldsymbol{\mu}_{2}\right)\right\}
\end{aligned}
$$

where $\pm \boldsymbol{\mu}_{1}$ and $\pm \boldsymbol{\mu}_{2}$ are axes angles, $\alpha$ apart, and $g(\cdot ; \pm \boldsymbol{\mu})$ is the probability density function of some axial distribution that has rotational symmetry about its mode $\pm \boldsymbol{\mu}$.

ii. The quadrimodal equal mixture model can be thought of intuitively as obtained by "pulling apart" a bimodal equal mixture distribution into two bimodal equal mixture distributions with planes angle $\gamma$ apart so that it has four equally strong modes. More precisely, the proba- 
Table 1. The $p$ values and corresponding decisions at the $5 \%$ significance level of randomisation tests of bimodality for bimodal equal mixtures of synthetic Watson distributions; $n$ is the total sample size and $B=999$ further randomisation samples per dataset (see text for details).

\begin{tabular}{|c|c|c|c|c|c|c|}
\hline \multirow{2}{*}{$\begin{array}{l}\text { True number } \\
\text { of modes }\end{array}$} & \multirow[b]{2}{*}{$\kappa$} & \multirow[b]{2}{*}{$n$} & \multicolumn{2}{|c|}{$S_{1}-S_{3}$ test } & \multicolumn{2}{|c|}{$S_{11}-S_{33}$ test } \\
\hline & & & $p$ value & No. of modes & $p$ value & No. of modes \\
\hline 2 & 10 & 52 & 0.37 & 2 & 0.51 & 2 \\
\hline 2 & 10 & 360 & 0.27 & 2 & 0.33 & 2 \\
\hline 2 & 20 & 52 & 0.66 & 2 & 0.69 & 2 \\
\hline 2 & 20 & 360 & 0.20 & 2 & 0.25 & 2 \\
\hline 2 & 50 & 52 & 0.45 & 2 & 0.48 & 2 \\
\hline 2 & 50 & 360 & 0.35 & 2 & 0.42 & 2 \\
\hline 2 & 100 & 52 & 0.34 & 2 & 0.41 & 2 \\
\hline 2 & 100 & 360 & 0.60 & 2 & 0.63 & 2 \\
\hline
\end{tabular}

Table 2. The $p$ values and corresponding decisions at the $5 \%$ significance level of randomisation tests of bimodality for quadrimodal equal mixtures of Watson distributions; $n$ is the total sample size and $B=999$ further randomisation samples per dataset (see text for details).

\begin{tabular}{lrrrr|rr}
\hline \multirow{2}{*}{$\begin{array}{l}\text { True number } \\
\text { of modes }\end{array}$} & & & \multicolumn{2}{c|}{$S_{1}-S_{3}$ test } & \multicolumn{2}{c}{$S_{11}-S_{33}$ test } \\
\cline { 5 - 7 } & $\kappa$ & $n$ & $p$ value & No. of modes & $p$ value & No. of modes \\
\hline 4 & 10 & 52 & 0.00 & $>2$ & 0.00 & $>2$ \\
4 & 10 & 360 & 0.00 & $>2$ & 0.00 & $>2$ \\
4 & 20 & 52 & 0.00 & $>2$ & 0.00 & $>2$ \\
4 & 20 & 360 & 0.00 & $>2$ & 0.00 & $>2$ \\
4 & 50 & 52 & 0.00 & $>2$ & 0.00 & $>2$ \\
4 & 50 & 360 & 0.00 & $>2$ & 0.00 & $>2$ \\
4 & 100 & 52 & 0.00 & $>2$ & 0.00 & $>2$ \\
4 & 100 & 360 & 0.00 & $>2$ & 0.00 & $>2$ \\
\hline
\end{tabular}

bility density is

$f_{4}\left( \pm \boldsymbol{x} ;\left\{ \pm \boldsymbol{\mu}_{1}, \pm \boldsymbol{\mu}_{2}\right\}, \gamma\right)=\frac{1}{4} \sum_{\varepsilon, \eta} g\left( \pm \boldsymbol{x} ; \pm \boldsymbol{\mu}_{\varepsilon, \eta}\right)$,

where

$\boldsymbol{\mu}_{\epsilon, \eta}=\check{c}\left(c \boldsymbol{v}_{1}+\epsilon s \boldsymbol{v}_{2}\right)+\eta \check{s} \boldsymbol{v}_{3}$,

with $\quad c=\cos (\alpha / 2), s=\sin (\alpha / 2), \check{c}=\cos (\gamma / 2), \check{s}=$ $\sin (\gamma / 2), \cos (\alpha)=\boldsymbol{\mu}_{1}{ }^{\prime} \boldsymbol{\mu}_{2}$ and $(\epsilon, \eta)$ runs through $\{ \pm 1\}^{2}$. If $\gamma=0$, then Eq. (3) reduces to Eq. (2).

The problem of interest is to decide whether the parent distribution is Eq. (1) or Eq. (2).

\subsubsection{The tests}

Given axes $\pm \boldsymbol{x}_{1}, \ldots \pm \boldsymbol{x}_{n}$ we denote by $\pm \hat{\boldsymbol{v}}_{1}$ and $\pm \hat{\boldsymbol{v}}_{3}$, respectively, the principal axes of the orientation tensor corresponding to the largest and smallest eigenvalues, $S_{1}$ and $S_{3}$. We can also define

$S_{11}=n^{-1} \sum_{i=1}^{n}\left(\hat{v}_{1}{ }^{\prime} \boldsymbol{x}_{i}\right)^{4}, S_{33}=n^{-1} \sum_{i=1}^{n}\left(\hat{v}_{3}{ }^{\prime} \boldsymbol{x}_{i}\right)^{4}$.

$S_{1}$ and $S_{3}$ are the second moments of $\pm \boldsymbol{x}_{1}, \ldots \pm \boldsymbol{x}_{n}$ along the first and third principal axes, respectively, whereas $S_{11}$ and
$S_{33}$ are the fourth moments along these principal axes. Therefore, both $S_{1}-S_{3}$ and $S_{11}-S_{33}$ are measures of the anisotropy of $\pm \boldsymbol{x}_{1}, \ldots \pm \boldsymbol{x}_{n}$.

Some algebra shows that

$T_{1}-T_{3}=\cos (\gamma)\left\{E\left[x^{2}\right]-E\left[v^{2}\right]\right\}$,

where $T_{1}$ and $T_{3}$ are the population versions of $S_{1}$ and $S_{3}$, respectively, and $\pm x$ and $\pm v$ are the components of $\pm \boldsymbol{x}$ in the quadrimodal equal mixture model Eq. (2) along its first and third principal axes, respectively. Then Eq. (4) gives

$\cos (\gamma) \approx \frac{S_{1}-S_{3}}{E\left[x^{2}\right]-E\left[v^{2}\right]}$,

and therefore it is sensible to

reject bimodality for small values of $S_{1}-S_{3}$.

Further algebra shows that

$T_{11}-T_{33}=\cos (\gamma)\left\{E\left[x^{4}\right]-E\left[v^{4}\right]\right\}$,

where $T_{11}$ and $T_{33}$ are the population versions of $S_{11}$ and $S_{33}$, respectively. Then Eq. (6) gives

$\cos (\gamma) \approx \frac{S_{11}-S_{33}}{E\left[x^{4}\right]-E\left[v^{4}\right]}$, 
Table 3. The $p$ values and corresponding decisions at the $5 \%$ significance level of randomisation tests of bimodality for natural datasets; $n$ is the total sample size and $B=999$ further randomisation samples per dataset (see text for details).

\begin{tabular}{lrrr|rr}
\hline Field & & \multicolumn{2}{c|}{$S_{1}-S_{3}$ test } & \multicolumn{2}{c}{$S_{11}-S_{33}$ test } \\
\cline { 3 - 6 } location & $n$ & $p$ value & No. of modes & $p$ value & No. of modes \\
\hline Gruinard & 75 & 0.00 & $>2$ & 0.00 & $>2$ \\
Chimney Rock & 86 & 0.99 & 2 & 1.00 & 2 \\
Flamborough & 346 & 0.00 & $>2$ & 0.00 & $>2$ \\
Central Italy & 1182 & 0.00 & $>2$ & 0.00 & $>2$ \\
Aegean & 156 & 0.00 & $>2$ & 0.00 & $>2$ \\
Tibet & 168 & 0.00 & $>2$ & 0.00 & $>2$ \\
\hline
\end{tabular}

and so it is sensible to

reject bimodality for small values of $S_{11}-S_{33}$.

The significance of tests (5) or (7) is assessed by comparing the observed value of the statistic with the randomisation distribution. This is achieved by creating further $B$ pseudosamples (for a suitable positive integer $B$ ), in each of which the $i$ th observation is obtained from $\pm \boldsymbol{x}_{i}$ by rotating $\pm x_{i}$ about the closer of the two fitted modes through a uniformly distributed random angle. The $p$ value is taken as the proportion of the $B+1$ values of the statistic that are smaller than (or equal to) the observed value.

\subsection{Results for synthetic datasets}

Table 1 gives the $p$ values and corresponding decisions (at the $5 \%$ level) obtained by applying the tests to some synthetic datasets simulated from the bimodal equal mixture model. Table 2 does the same for some datasets simulated from the quadrimodal equal mixture model. In each case, both tests come to the correct conclusion.

\subsection{Results for natural datasets}

Table 3 gives the $p$ values and corresponding decisions (at the $5 \%$ level) obtained by applying the tests to the natural datasets discussed in Sect. 3.2. For each dataset, the two tests come to the same conclusion, which is plausible in view of Fig. 5. Figure 7 shows the fabric eigenvalue plot for these datasets.

\section{Discussion}

In the analysis described above and the tests we performed with synthetic datasets, we assumed that bimodal and quadrimodal Watson orientation distributions provide a reasonable approximation to the distributions of poles to natural fault planes. In terms of the underlying statistics this is unproven, but we know of no compelling evidence in support of alternative distributions. New data from carefully controlled laboratory experiments on rock or analogous materials might

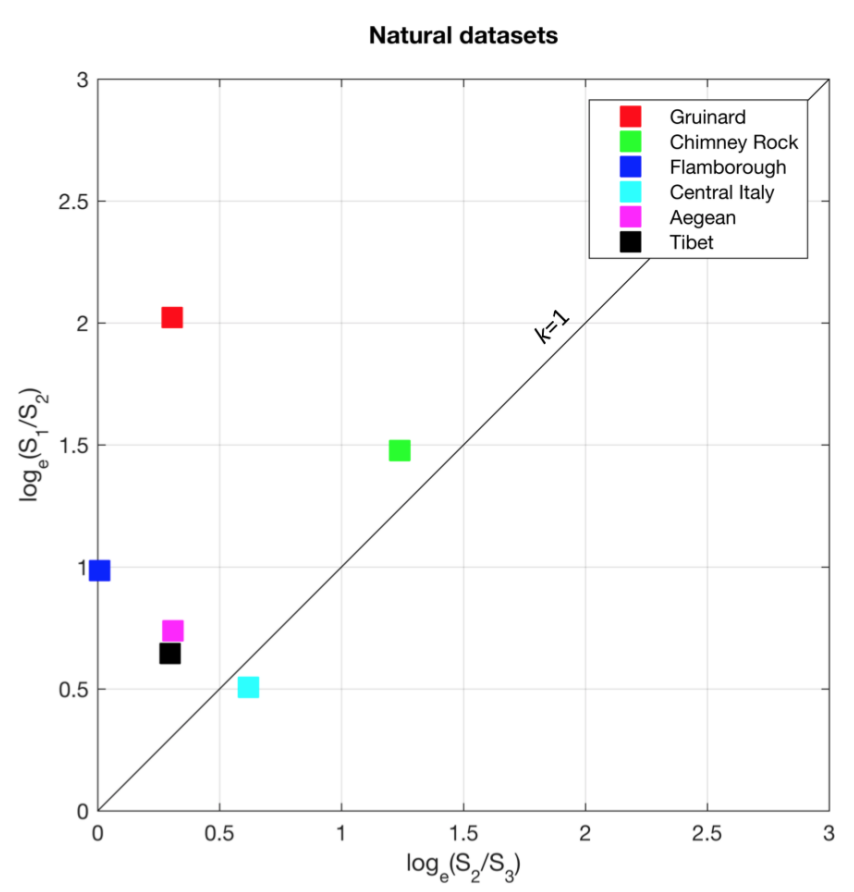

Figure 7. Eigenvalue ratio plot for the natural datasets shown in Fig. 5. All but one dataset (central Italy) lie above the line for $k=1$. The best-constrained quadrimodal fault dataset (Gruinard) has the highest ratio of $\log _{e}\left(S_{1} / S_{2}\right)$.

provide important constraints for the underlying statistics of shear fracture plane orientations.

We have tested our new methods on synthetic and natural datasets. Arguably, six natural datasets are insufficient to establish firmly the primacy of polymodal orthorhombic fault patterns in nature (Fig. 7). However, we reiterate the key recommendation from Healy et al. (2015): to be useful for this task, fault orientation datasets need to show clear evidence of contemporaneity among all fault sets through tools such as matrices of cross-cutting relationships (Potts and Reddy, 2000). In addition, as shown above, larger datasets $(n>200)$ tend to show clearer patterns. Scope exists to collect fault or shear fracture orientation data from sources other than outcrops: Yielding (2016) has measured normal faults in seismic 

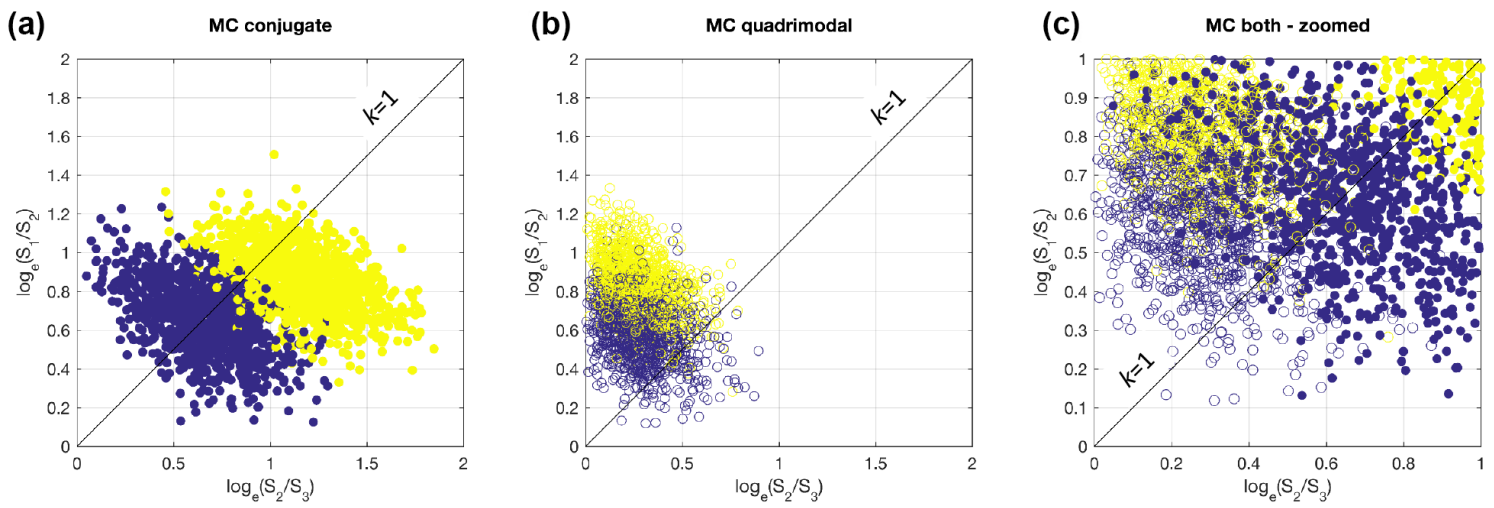

Figure 8. Eigenvalue ratio plots of synthetic data to illustrate the impact of dispersion on the ability of this plot to discriminate between conjugate (bimodal) and quadrimodal fault data. (a) Monte Carlo ensemble of 2000 conjugate fault populations (mixtures of two equal Watson distributions), with $\kappa$ varying from 5 (dark blue) to 10 (yellow). (b) Monte Carlo ensemble of 2000 quadrimodal fault populations (mixtures of four equal Watson distributions), with $\kappa$ varying from 5 (dark blue) to 10 (yellow). (c) Data from (a) and (b) merged onto the same plot and enlarged to show the region close to the origin. Note the considerable overlap between the conjugate (bimodal) data with the quadrimodal data, especially for $\kappa=5$ (dark blue).
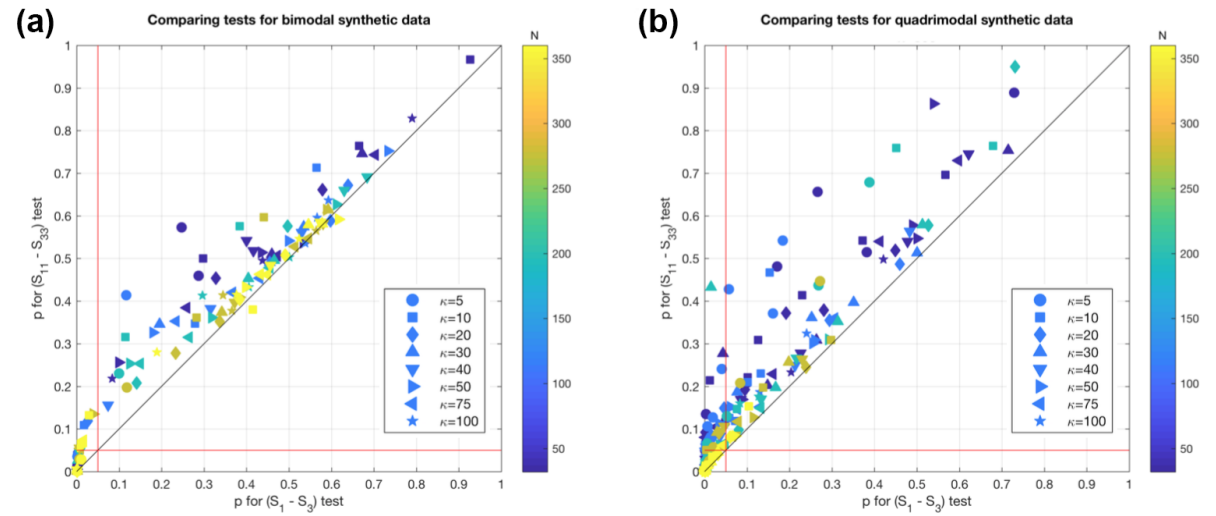

Figure 9. Eigenvalue ratio plots comparing the relative performance of the two tests proposed in this paper. The red lines denote $p$ values for either test at $p=0.05$, and the diagonal black line is the locus of points where $p\left(S_{1}-S_{3}\right)=p\left(S_{11}-S_{33}\right)$. (a) For bimodal synthetic datasets with size $(n)$ varying from 32-360 and concentration $(\kappa)$ varying from 5-100, both tests perform well and reject the majority of the datasets ( $p \gg 0.05$ ). The $p$ values for the $S_{11}-S_{33}$ test are, on average, slightly higher than those for the $S_{1}-S_{3}$ test across a range of dataset sizes and concentrations. (b) For quadrimodal synthetic datasets, many of the $p$ values are $<0.05$, and this is especially true for the larger datasets (higher $n$, green-yellow). Smaller datasets (blue) can return $p$ values $>0.05$.

reflection data from the North Sea and Ghaffari et al. (2014) measured faults in centimetre-sized samples deformed in the laboratory and then scanned by X-ray computerised tomography.

The Chimney Rock dataset is probably not orthorhombic according to the two tests and lies close to the line for $k=1$ in Fig. 7. It is interesting to note that the Chimney Rock data, and other fault patterns from the San Rafael area of Utah, are considered as displaying orthorhombic symmetry by Krantz (1989) and Reches (1978). However, a subsequent reinterpretation by Davatzes et al. (2003) has ascribed the fault pattern to overprinting of earlier deformation bands by later sheared joints. This may account for the inconsistent results of our tests when compared to the position of the pattern on the eigenvalue plot. The central Italy dataset (taken from Roberts, 2007) is very large $(n=1182)$ and the data were measured over a wide geographical area. The dataset lies below the line for $k=1$ on the fabric eigenvalue plot (Fig. 7), which might suggest it is bimodal. However, for fault planes measured over large areas there is a significant chance that regional stress variations may have produced systematically varying orientations of fault planes.

A final point concerns dispersion (noise) in the data. Synthetic datasets of bimodal (conjugate) and quadrimodal patterns with low values of $\kappa$, the Watson concentration parameter, fall into overlapping fields on the eigenvalue fabric plot. We ran 1000 Monte Carlo simulations of bimodal and quadrimodal Watson distributions each with $n=52$ poles and 
$\kappa=5$ and 10, and the results are shown in Fig. 8. Bimodal (conjugate) datasets for these dispersed and sparse patterns lie across the $1: 1$ line on the fabric plot (Fig. $8 \mathrm{a} ; \kappa=5$ in blue, $\kappa=10$ in yellow). Quadrimodal datasets for these parameters are also noisy, with some fabrics lying below the $1: 1$ line (Fig. 8b; $\kappa=5$ in blue, $\kappa=10$ in yellow). Under these conditions of low $\kappa$ (dispersed) and low $n$ (sparse), it can be difficult to separate bimodal (conjugate) from quadrimodal fault patterns. However, we assert that this may not matter: a noisy and dispersed "bimodal" conjugate fault pattern is in effect similar to a polymodal pattern; i.e. slip on these dispersed fault planes will produce a bulk 3-D triaxial strain.

To assess the relative performance of the two tests presented in this paper, we generated synthetic bimodal and quadrimodal distributions and compared the resulting $p$ values from applying both the $S_{1}-S_{3}$ and $S_{11}-S_{33}$ tests to the same data. The results are shown in Fig. 9, displayed as cross-plots of $p\left(S_{1}-S_{3}\right)$ versus $p\left(S_{11}-S_{33}\right)$. While there is a slight tendency for the $p$ values from the $S_{11}-S_{33}$ test to exceed those of the $S_{1}-S_{3}$ test (i.e. the points tend on average to plot above the $1: 1$ line), neither of the tests can be said to be "better" or more "accurate". We therefore recommend the $S_{1}-S_{3}$ test as simpler and sufficient.

\section{Summary}

Bimodal (conjugate) fault patterns form in response to a bulk plane strain with no extension in the direction parallel to the mutual intersection of the two fault sets. Quadrimodal and polymodal faults form in response to bulk triaxial strains and constitute the more general case for brittle deformation on a curved Earth (Healy et al., 2015). In this contribution, we show that distinguishing bimodal from quadrimodal fault patterns based on the orientation distribution of their poles can be achieved through the eigenvalues of the secondand fourth-rank orientation tensors. We present new methods and new open source software written in $\mathrm{R}$ to test for these patterns. Tests on synthetic datasets in which we controlled the underlying distribution to be either bimodal (i.e. conjugate) or quadrimodal (i.e. polymodal, orthorhombic) demonstrate that a combination of fabric eigenvalue (modified Flinn) plots and our new randomisation tests can succeed. Applying the methods to natural datasets from a variety of extensional normal-fault settings shows that five out of the six fault patterns considered here are probably polymodal. The most tightly constrained natural dataset (Gruinard) displays clear orthorhombic symmetry and is unequivocally polymodal. Most map-scale natural faults evolve and grow through interaction, splaying and coalescence, and in some cases through reactivation under stress rotation. Variation within fault orientation datasets is therefore inherent. Statistical tests can help to discern this variation and guide the interpretation of any underlying pattern. We encourage other workers to apply these tests to their own data, assess the symmetry in the brittle fault pattern and to consider what this means for the causative deformation.

Data availability. Datasets used in this study are available from the first author on request (d.healy@abdn.ac.uk).

Author contributions. DH devised the study, and PJ formulated the statistical tests and wrote the R code. DH collated the data and wrote the paper with input from PJ.

Competing interests. The authors declare that they have no conflict of interest.

Acknowledgements. David Healy gratefully acknowledges receipt of NERC grant NE/N003063/1 and thanks the School of Geosciences at the University of Aberdeen for accommodating a period of research study leave, during which time this paper was written. We thank two anonymous reviewers, plus Atilla Aydin (Stanford) and Nigel Woodcock (Cambridge), for comments which helped us improve the paper.

Edited by: Federico Rossetti

Reviewed by: Nigel Woodcock, Atilla Aydin, and two anonymous referees

\section{References}

Aydin, A. and Reches, Z. E.: Number and orientation of fault sets in the field and in experiments, Geology, 10, 107-112, 1982.

Blenkinsop, T. G.: Relationships between faults, extension fractures and veins, and stress, J. Struct. Geol., 30, 622-632, 2008.

Davatzes, N. C., Aydin, A., and Eichhubl, P.: Overprinting faulting mechanisms during the development of multiple fault sets in sandstone, Chimney Rock fault array, Utah, USA, Tectonophysics, 363, 1-18, 2003.

Ekström, G., Nettles, M., and Dziewoński, A. M.: The global CMT project 2004-2010: Centroid-moment tensors for 13017 earthquakes, Phys. Earth Planet. Int., 200, 1-9, 2012.

Fisher, N. I., Lewis, T., and Embleton, B. J.: Statistical analysis of spherical data, Cambridge University Press, 1987.

Flinn, D.: On folding during three-dimensional progressive deformation, Q. J. Geol. Soc., 118, 385-428, 1962.

Ghaffari, H. O., Nasseri, M. H. B., and Young, R. P.: Faulting of Rocks in a Three-Dimensional Stress Field by Micro-Anticracks, Sci. Rep., 4, 5011, https://doi.org/10.1038/srep05011, 2014.

Healy, D., Jones, R. R., and Holdsworth, R. E.: Three-dimensional brittle shear fracturing by tensile crack interaction, Nature, 439, 64-67, 2006a.

Healy, D., Jones, R. R., and Holdsworth, R. E.: New insights into the development of brittle shear fractures from a 3-D numerical model of microcrack interaction, Earth Planet. Sc. Lett., 249, 1428, 2006b. 
Healy, D., Blenkinsop, T. G., Timms, N. E., Meredith, P. G., Mitchell, T. M., and Cooke, M. L.: Polymodal faulting: Time for a new angle on shear failure, J. Struct. Geol., 80, 57-71, 2015.

Krantz, R. W.: Orthorhombic fault patterns: the odd axis model and slip vector orientations, Tectonics, 8, 483-495, 1989.

Lisle, R. J. and Leyshon, P. R.: Stereographic projection techniques for geologists and civil engineers, Cambridge University Press, 2004.

Mardia, K. V. and Jupp, P. E.: Directional statistics, John Wiley \& Sons, 2000.

McCormack, K. D. and McClay, K. R.: Orthorhombic faulting in the Beagle Sub-basin, North West Shelf, Australia, Geological Society, London, Special Publications, 476, 2018.

Peacock, D. C. P. and Sanderson, D. J.: Effects of layering and anisotropy on fault geometry, J. Geol. Soc., 149, 793-802, 1992.

Potts, G. J. and Reddy, S. M.: Application of younging tables to the construction of relative deformation histories -1 : fracture systems, J. Struct. Geol., 22, 1473-1490, 2000.

R Core Team: R: A language and environment for statistical computing. R Foundation for Statistical Computing, Vienna, Austria, available at: https://www.R-project.org/ (last access: 17 August 2018), 2017.
Ramsay, J.: Folding and fracturing of rocks, McGraw Hill Book Company, 568 pp., 1967.

Reches, Z. E.: Analysis of faulting in three-dimensional strain field, Tectonophysics, 47, 109-129, 1978.

Reches, Z. E.: Faulting of rocks in three-dimensional strain fields II. Theoretical analysis, Tectonophysics, 95, 133-156, 1983.

Reches, Z. E. and Dieterich, J. H.: Faulting of rocks in threedimensional strain fields I. Failure of rocks in polyaxial, servocontrol experiments, Tectonophysics, 95, 111-132, 1983.

Roberts, G. P.: Fault orientation variations along the strike of active normal fault systems in Italy and Greece: Implications for predicting the orientations of subseismic-resolution faults in hydrocarbon reservoirs, AAPG Bull., 91, 1-20, 2007.

Scheidegger, A. E.: On the statistics of the orientation of bedding planes, grain axes, and similar sedimentological data, US Geological Survey Professional Paper, 525, 164-167, 1965.

Woodcock, N. H.: Specification of fabric shapes using an eigenvalue method, Geol. Soc. Am. B., 88, 1231-1236, 1977.

Yielding, G.: The geometry of branch lines, in: The Geometry and Growth of Normal Faults, edited by: Childs, C., Holdsworth, R. E., Jackson, C. A.-L., Manzocchi, T., Walsh, J. J., and Yielding, G., Geological Society, London, Special Publications, 439, 2016. 